\title{
A SIMPLE INSTRUMENTATION SYSTEM FOR SEPARATION OF WHOLE BLOOD COMPONENTS USING CENTRIFUGE TECHNIQUE AND MEASUREMENT OF RBC
}

\author{
Dodda Basavanagoud B ${ }^{1}$, K. Padma Priya ${ }^{2}$ \\ ${ }^{1}$ Professor, Dept. of ECE, RYM Engineering College, Bellary-583104, Karnataka, India \\ ${ }^{2}$ Associate Professor, Dept. of ECE, JNTUK College of Engineering, Vizianagaram-535002, Andhra Pradesh, India
}

\begin{abstract}
Development of tiny portable, cost effective simple Instrumentation embedded system will help in medical field for the treatment of adult patients. The need of embedded system is more common now days in medical field. Most of the annual medical check-ups involve the physician ordering only routine blood tests. With blood test results in hand, one can catch critical changes in the body before they manifest as heart disease, cancer, diabetes, or worse. According to recent surveys, it is very essential for adults to checkup blood to know the hemoglobin, platelets, plasma contents etc. to avoid risks in life. Even rural common people need to come for cities to checkup blood. This work mainly focuses on design of such low cost embedded solution for the measurement of blood components especially for adults. The people may get rough idea about their health using this low cost device. We have used centrifuge technique in our system which works on the principle of sedimentation for the separation of blood components. The low power ARM7TDMI LPC2148 microcontroller is used in our system. The cost of the system is affordable even for common people
\end{abstract}

Keywords: Centrifuge technique, ARM7 LPC2148, Portable, DC motor, PWM, Blood components, RBC, WBC

$* * *$

\section{INTRODUCTION}

The need for low cost embedded system is very essential in medical field to design cost effective biomedical instrumentation systems. Now-a-days the use of embedded systems in the life science industry is of great interest since it allows the creation of handy/fieldable medical examining systems. Even processing power of such microcontrollers is good enough to process the parameters. Due to small sizes and functionality requirements needed specificity in function, embedded systems are widely used in medical applications. Embedded systems play key role in major medical equipment such as CT scanners, MRI scanners, ECG, pacemakers and implanted pumps, implanted heart monitors, blood measurement instruments, infusion pumps, glucose measuring device, respiratory ventilators, anesthetic machines, endoscopy machines, spirometer, defibrillators, ENT workstations etc. Blood is a valuable source to get various information about our whole body. Though the elements of blood are usually keen within the constant range, the changes take place when abnormalities are found in our bodies.

Therefore, when blood is examined, not only the sickness of blood but also various information on our bodies is obtained. Most of the rural parts of our country still have insufficient equipment for examining the blood components. Even if we have, however, it takes about one week time the results are obtained, when inspecting in small medical agency. By the blood examination, it is possible to detect a lot of diseases such as the lifestyle diseases an treat it early stage, and many people receive the inspection for sick prevention and a healthy check in recent years. Hence, with the availability of low cost and low power microcontrollers it is possible for us to develop rapid and tiny portable systems for the treatment of patients especially for the rural people. Blood examining system design consists of two major parts, blood component separation and their detection. There are several techniques used in practice for the separation of blood components $[1,3,6,8]$.

\subsection{Optical Separation}

This method uses the recent advances in manipulation of optical tweezers. Optical tweezers [1] is a manipulation tool developed by Ashkin, where a tightly focused single laser beam is used to trap a single particle. Optical tweezers have been widely used since then to trap single particles in micro fluidic systems. Advances in optical manipulation allowed the creation of threedimensional arrays of light traps (optical lattices) using holographic optical tweezers, generalized phase contrast or diffractive optical element and multi-beam interferences.

\subsection{Magnetic Separation}

The concept of a conventional magnetic separation device is a straightforward and long-established process $[1,3]$ a magnet is placed in the vicinity of a column containing the cells to be 
separated. Magnetically labeled cells are retained in the column, whereas non-labeled cells will be flushed with the buffer allowing the immunological separation of species. The column is removed from the magnet in a second step and flushed to allow the collection of the sorted particles. This kind of separation is termed magnetic activated cell sorting (MACS). MACS are also a method patented by Miltonic Biotec but are widely available commercially under other names from manufacturers such as Dexter or Stem Cell.

\subsection{Electrical Separation}

Originally, most of the first separation techniques for micro particles used electrical forces. Indeed, electricfield- based manipulation is well suited at the microscale because of the ease with which high electric fields can be produced with micron size gaps and voltage of several volts only [1]. There are two main types of electric field-based manipulation, depending on the properties of the particles to be sorted. Electrophoresis, the movement of charged particles in a uniform electric field, is a very well known technique to separate and transport different kinds of charged particles.

\subsection{Centrifugal Technique}

A laboratory centrifuge is a piece of laboratory equipment, driven by a motor, which spins liquid samples at high speed. There are various types of centrifuges, depending on the size and the sample capacity.

Referring to different types of continuous and non-continuous separation techniques $[3,9,10,11,17]$, we have been motivated to develop a portable, low cost, fieldable, battery operated instrumentation system for separation of whole blood components using centrifugal technique.

\section{WHOLE BLOOD SEPERATION TECHNIQUE}

Centrifugal technique has been used in our model for separation of blood components. Like all other centrifuges, laboratory centrifuges work by the sedimentation principle, where the centripetal acceleration is used to separate substances of greater and lesser density. Setup which spins the blood sample is as shown in fig- 1 .

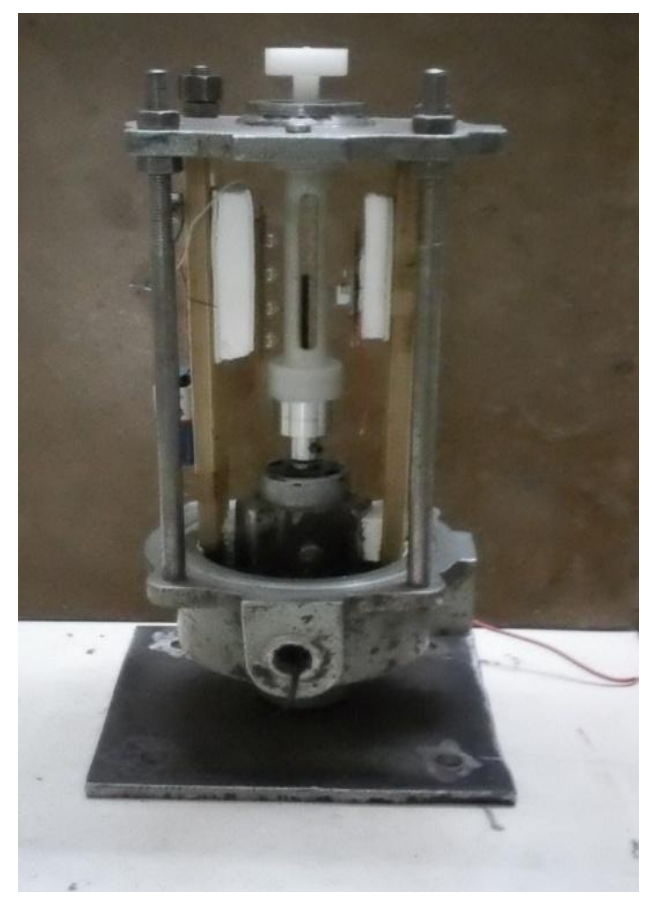

Fig- 1: Centrifuge system used for blood components separation

Mainly DC motor with voltage $12 \mathrm{~V}$ and current $3.3 \mathrm{amps}$ rating is used for the centrifuge system design. The motor is rotated with a speed of $10000 \mathrm{rpm}$ for a time period of 5 minutes to separate the blood components, which has been placed in capillary tube.

\subsection{Photometric System}

Most photometers detect the light with photo resistors, photodiodes or photomultipliers. To analyze the matter presented in the sample, the photometric system measure the light after it has passed through sample. The amount of light absorption, scattering, and reflection by the sample is the measure for the properties of the sample. Hence, once the whole blood is separated in to different blood components by centrifugal system, it is examined by a photometric system. In our system, 450-500 nm wavelength LED array is used as a light source, which is fixed at one side of the capillary tube along with a slit mechanism. Slit arrangement, which is made on the LED source array, allows the sample to expose to the narrowed beam of light. Depending upon the density of the components, the intensity of light at other end will varies. The light transmission/absorption by the sample is measured by the photo detector which is place on the same axis but the opposite side of the light source. TSL257 is a high-sensitivity low-noise light-to-voltage optical converter IC, which is fixed at the other end of the capillary tube, used as a photo detector. Photo detectors output is further acquired by the ARM through its onchip available A/D converter. 


\section{EXPERIMENTAL IMPLEMENTATION}

Block diagram of the system implementation for separation and detection of the whole blood components is as shown in fig-2.

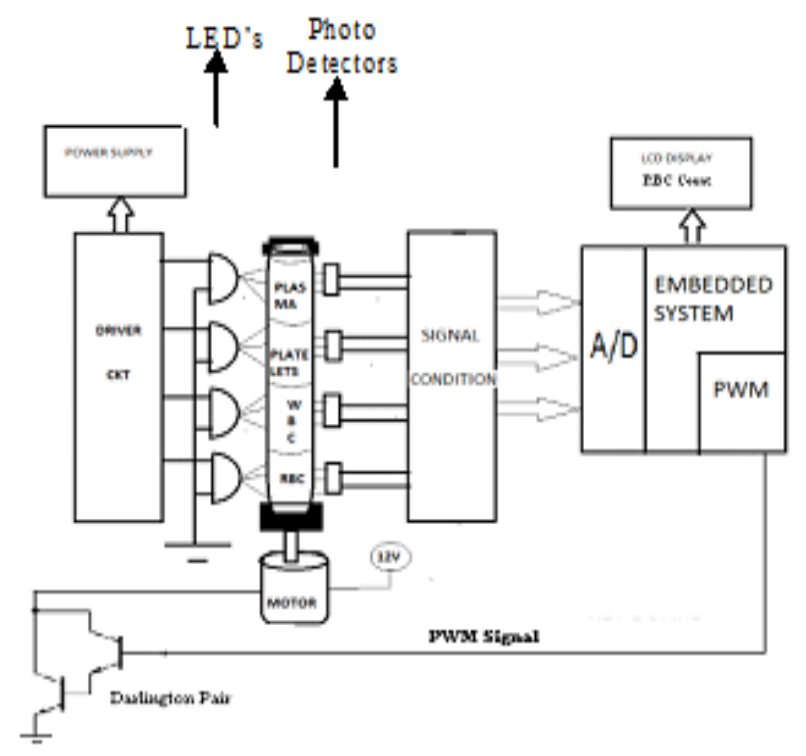

Fig-2: Experimental Block Diagram

Following are the major components which have been used in our system,

\subsection{Motor Drive}

DC motor operated with +12 Volts is the key element of the centrifugal system, and PWM power controlled mechanism is used to control the speed of the motor. A PID control algorithm has been developed to generate necessary on-chip PWM control signals to the power drive of the DC motor. A simple Darlington pair amplifier is used as a power actuator. The DC motor is rotated with a speed of $10000 \mathrm{RP} \mathrm{M}$ for a period of 5 to 10 minutes, so that the whole blood gets separated into different component layers like RBC, WBC, Platelets and Plasma.

\subsection{Photo Detectors}

The TSL257 is a high-sensitivity low-noise light-to-voltage optical converter that combines a photodiode and a transimpedance amplifier on a single monolithic CMOS integrated circuit is used as a detector in the system [12]. Output voltage of the detector is proportional to the light intensity (irradiance) which is falls on the photodiode. The TSL257 has a transimpedance gain of $320 \mathrm{M} \Omega$. The device has improved offset voltage stability and low power consumption and is supplied in a 3-lead clear plastic side looker package with an integral lens.
Depending upon the volume of the components in the blood, the intensity will vary. These detectors are placed as a column on one side of the sample holder. On the opposite side, LED light sources are placed. The analog output from the detectors is further acquired by the ARM processor through its on-chip A/D converter.

\subsection{Embedded Processor}

ARM stands for Advanced RISC Machines. It is a 32 bit processor core used for high end applications.

The LPC2148 microcontrollers are based on a 16-bit/32-bit ARM7TDMI-S CPU with real-time emulation and embedded trace support with embedded high speed flash memory ranging from 32KB to 512KB and TDMI_S can expands.

ARM7LPC2148 controller is used for signal acquisition, processing and displaying the results and it is key a element in the present system design. LPC2148 is ideal for hand help medical diagnosing systems because of its tiny size, low power consumption and low in cost as compare to its same class of embedded systems. Further, ARM7LPC2148 allows to upgrade the system for the future use as it has various inbuilt peripherals such as 32-bit timers, single or dual 10-bit ADC(s), 10-bit DAC, PWM channels, 45 fast GPIO lines with up to nine edge or level sensitive external interrupts, full speed USB 2.0, multiple UARTs, SPI, I2C bus and etc.

In the present system, on-chip PWM channel is programmed to control the speed of the centrifuge system. Six on-chip ADC channels are used to acquire the signal from the detectors. Then, the data is processed and displayed RBC count in millions/micro-litre.

A low cost and compact 16X2 Liquid Crystal Display (LCD) is used as the medium for displaying RBC count value.

A 128-bit wide memory interface and unique accelerator architecture enable 32-bit code execution at the maximum clock rate, for critical code size applications. The alternative 16-bit Thumb mode reduces code by more than $30 \%$ with minimal performance penalty. The LPC 2148 microcontroller is shown in fig-.3.

\section{EXPERIMENTAL RESULTS}

The entire set up made for the separation and detection of the whole blood components is as shown in fig. 3 . 


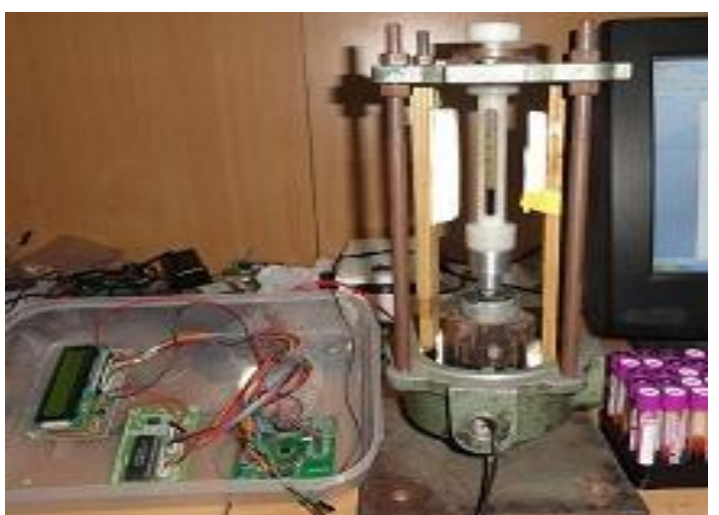

Fig-3: Separation \& Detection Experimental Setup

The sample holder containing whole blood is spinned for a period of 5 minutes with a speed of 10000 RPM to divide into individual components. Fig- 4 shows the results of sample column separated into individual components. Since RBC contains some heavy particles such as iron, it settles down at the bottom of the capillary tube (can be seen as red in color) above which WBC cells get accumulated (can be seen as white in color), then the platelets (can be seen as a yellow circle on top of the WBC). Rest all the plasma.

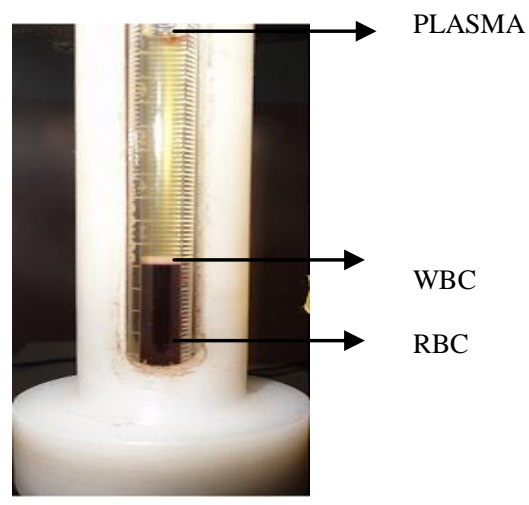

Fig- 4: A blood sample column after the separation

The resultant sample column is exposed to a photometric system that measures the RBC component and displays the count in terms of millions/micro-liter. Table 1 shows the experimental results of RBC count Vs A/D converter readout by the ARM processor.

Table-1: RBCVs A/D readout

\begin{tabular}{|l|l|l|}
\hline Parameter & Count & A/D readout \\
\hline RBC & $\begin{array}{l}\text { 3.5. to } 7.5 \text { million } \\
\text { cells } / \mu \text { liter }\end{array}$ & 1.25 to $0.33 \mathrm{~V}$ \\
\hline
\end{tabular}

RBC count Vs ADC voltage graph is shown in the fig -5 . Results are drawn after calibrating photometric system with the standard hematology analyzer. The voltage obtained corresponding to $\mathrm{RBC}$ is substituted in a calibrated equation, so that we get the cell count.

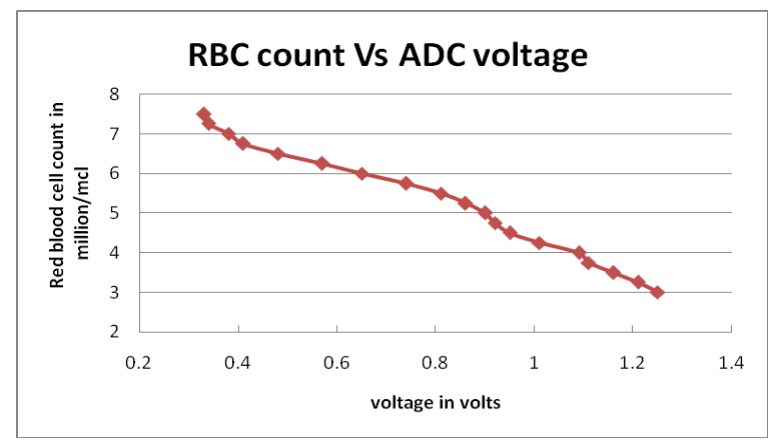

Fig-5: RBC count Vs ADC Voltage Plot

\section{CONCLUSIONS}

It can be concluded that, system is having $95 \pm 04 \%$ repeatability for same samples and the present system readings are compared with standard cell counter readings.

In our existing system the time taken for blood separation takes almost 5-6 minutes, sample volume is $5 \mathrm{ml}$ and accuracy range is having $85 \pm 5 \%$.

\section{FUTURE WORK}

The major important parameters to be considered for an efficient system are the time, sample, cost, and accuracy. In our future work by keeping these we would like to measure WBC and plasma component. The major important parameters to be considered for an efficient system are the time, sample volume, and accuracy. Hence the system is intended to optimize in terms of time, sample volume, and accuracy.

\section{ACKNOWLEDGEMENTS}

We would like to thank Vijayanagar Institute of Medical Sciences (VIMS), Bellary, Karnataka for their support in providing blood samples and accessing their labs in order to calibrate our photometric system.

\section{REFERENCES}

[1]. M. Kersaudy-Kerhoas, R.Dhariwal, M.P.Y. Desmulliez:" Recent advances in micro particle continuous separation", IET Nanobiotech., 2007.

[2]. S.Shin, M.S Park, J.H Jang, Y.H.Ku and J.S Suh, "Measurement of red blood cell aggregation by analysis of light transmission in a pressure driven slit flow system", KoreaAustralia Rheology Journal, Sept. 2004, Vol. 16, No.3, pg 129134.

[3]. Dodda Basavana Goud B, K Padma Priya, Nagabhushan Katte, "A Review of Recent Advances in Separation and Detection of Whole Blood Components", World Journal of 
Science and Technology 2012, 2(5):05-09, ISSN: $2231-2587$. Available Online: www.worldjournalofscience.com

[4]. Katsutoshi OOE, Toshio Fukuda,et al 'Development of Micro Particles Separation Device with Piezo-Ceramic Vibrator', IEEE-2009.

[5]. David W. Inglis. "Efficient Microfluidic particle sepration arrays" Department of Physics, Macquaric University Sydney, NSW, 2109, Australia September 29, 2008

[6]. Hideaki Tsutsui, Chih-Ming Ho. "Cell Separation by noninertial force fields in microfluidic systems ". Mechnics research Communications 36(2009) 92-103

[7]. K.H. Han and A.B. Frazier. "Paramagnetic Capture Mode Magnetophoretic Microseparator for Blood Cells" IEE Proc.Nanobiotechnol Vol. 153 No 4, August-2006

[8]. Ae Gyoung Oh, Dong Woo Lee, and Young-Ho Cho. Korea. "A Continuous Cell Separator Based On Buoyant Force in Dissimilar Density Fluid Flows". IEEE-Digital Nanolocomotion Center -2010

[9]. Taotao Zhu, Francisco Marrero and Leidong Mao. "Continuous Separation of non magnetic particles through negative magnetophoresis inside Ferrofluids" Proceedings of IEEE-international Conference on Nano / Micro Engineered \& Molecular systems - 2010 Pg 1006-1011

[10]. S.S Kuntaegowdanahalli, A.A.S Bhagat and I.Papautsky "Continuous multi particle separation using deterministic focusing in spiral microchannels". Transducers - 2009, Pg.2139-2142

[11]. Siyang Zheng, Yu-Chong Tai and Harvey Kasdan "A micro device for separation of Erythrocytes and Leukocytes in human blood". Proceedings of the 2005 IEEE-Engineering in medicine and biology 27th conference - 2005, Pg 1024-1027

[12]. TSL257 - high-sensitivity low-noise light-to-voltage optical converter IC Data sheets on "http://www.taosinc.com " (Texas advanced optoelectronic Solutions)

[13]. LPC2148 Education Board-User's Guide on http://www.embeddedartists.com/ products/education/edu_2148.php

[14]. User Manual for CP-JR ARM7 USB-LPC2148/EXP on http://www.ett.co.th/ download2.html

[15]. UM10139 LPC214x User manual on http://www.nxp.com/documents/user_manual/UM10139.pdf 